\title{
Numerical study of a chemical method applied to instantaneous heat removal under high heat flux
}

\author{
Jing Li $\cdot$ Gao-Ming $\mathrm{He} \cdot$ Cheng Zeng $\cdot$ \\ Ye-Ming Liu
}

Received: 1 November 2012/ Accepted: 15 May 2013/Published online: 28 May 2013

(c) The Author(s) 2013. This article is published with open access at Springerlink.com

\begin{abstract}
In this study, a method of chemical cooling is put forward, that is, $\mathrm{C}-\mathrm{CO}_{2}$ endothermic reaction is applied to instantaneous heat removal under high heat flux. A method in which theoretical research is in combination with numerical simulation is used to study $\mathrm{C}-\mathrm{CO}_{2}$ endothermic reaction. In comparison with the theoretically computational results, numerical code is validated. A high heat flux of $500 \mathrm{~W} / \mathrm{cm}^{2}$ is applied to the research of the heat dissipation characteristics of $\mathrm{C}-\mathrm{CO}_{2}$ endothermic reaction. The theoretical calculation results show that, under a certain temperature and pressure condition, the $\mathrm{C}-\mathrm{CO}_{2}$ chemical endothermic reaction could remove heat from the system promptly; the product $\mathrm{CO}$ could be used as a supplementary medium of power source for cycling. Compared with water phase change, the $\mathrm{C}-\mathrm{CO}_{2}$ endothermic reaction appears to have stronger heat removal ability. "Species Transport" module in FLUENT was adopted to simulate the reaction. Under the same temperature and pressure condition, the numerical simulation results are found to be well congruous with theoretical results. The $\mathrm{C}-\mathrm{CO}_{2}$ endothermic reaction could make a high temperature in the reaction system due to a high heat
\end{abstract}

J. Li (凹) · G.-M. He · C. Zeng · Y.-M. Liu

Key Laboratory of Enhanced Heat Transfer and Energy

Conservation, Ministry of Education, South China University of

Technology, Guangzhou, People's Republic of China

e-mail: 1jing@scut.edu.cn

G.-M. He

e-mail: hegaoming1501@163.com flux reduce to a low temperature (below zero) promptly. The heat removal and reaction time are in consistence with theoretical calculation.

\section{List of symbols}

A Pre-exponential factor (1/s)

$c \quad$ Reactant concentration $\left(\mathrm{mol} / \mathrm{m}^{3}\right)$

$C_{p} \quad$ Heat capacity $(\mathrm{J} / \mathrm{kg} \mathrm{K})$

$D_{I, m} \quad$ Mass diffusivity of gas I in gas mixture $\left(\mathrm{m}^{2} / \mathrm{s}\right)$

Ea Activation energy (kJ/mol)

$m \quad$ Mass $(\mathrm{kg})$

$n \quad$ Reaction order (-)

$P \quad$ Pressure $(\mathrm{Pa})$

$q \quad$ Volumetric heat source $\left(\mathrm{W} / \mathrm{m}^{3}\right)$

$Q \quad$ Heat $(\mathrm{KJ})$

$R \quad$ Gas constant $(\mathrm{J} / \mathrm{mol} \mathrm{K})$

$S_{m} \quad$ Mass source term

$t \quad$ Time

$T$ Temperature (K)

$u, v \quad$ Velocities in axial and radial directions, respectively $(\mathrm{m} / \mathrm{s})$

$U \quad$ Velocity vector in the $\mathrm{x}$-direction and $\mathrm{y}$-direction, $\mathrm{u}$ and $\mathrm{v}$

$x \quad$ Coordinate in axial direction (m)

$y \quad$ Radial coordinate $(\mathrm{m})$

$Y \quad$ Mass fraction $(\%)$

\section{Greek letters}

$\kappa$ Reaction rate constant

$\theta$ Surface fraction

$\beta$ Absorption capacity

$\gamma \quad$ Reaction rate

$\rho$ Density $\left(\mathrm{kg} / \mathrm{m}^{3}\right)$

$\mu$ Dynamic viscosity $(\mathrm{kg} / \mathrm{m} * \mathrm{~s})$

$\lambda$ Thermal conductivity $\left(\mathrm{W} / \mathrm{m}^{*}{ }^{\circ} \mathrm{C}\right)$ 


\section{Introduction}

With the development of new high-techs, occasions due to high and even ultra high heat flux appear. High heat flux results in high temperatures, which need higher requirements for the property of material and operating conditions and produce a negative effect on the service life-span of material. So heat removal problem caused by dense situation of high heat flux becomes increasingly serious. For example, ablation on the electrode surface caused by high heat flux, not only results in degradation of the overall performance of equipment, but also produces a negative effect on the service lifespan of equipment [1,2]; in order to improve the thermal efficiency and power output, advanced gas turbine engines usually work under high temperatures of $1,300-1,500{ }^{\circ} \mathrm{C}$, which cause the operating temperature of turbine blade to be much higher than that of metal materials allowable temperature, so reasonable cooling for blade becomes a problem which we have to be faced with [3]; heat flux density on the thermal deposit surface of high power semiconductor laser can reach as high as $400 \mathrm{~W} / \mathrm{cm}^{2}$, which is limited to a certain level of performance and further improvement by heat removal problem [4]; temperature of hypersonic aircraft (Mach number is 8 or above) head can reach $2,300{ }^{\circ} \mathrm{C}$, and combustion indoor temperature can reach above $3,000{ }^{\circ} \mathrm{C}$ [5].

Some mature forced cooling methods at present are based on physical heat sink, including forced air cooling, water phase change, pool boiling, spray cooling, microchannel cooling and micro-jet array cooling et al.

Forced air cooling [6] is a physical cooling method which uses the fan to blast or ventilate to improve air flow rate to achieve the purpose of cooling. It is widely used in high-power electronic device field, because its heat transfer capacity is several times more than that of natural air cooling, it is, however, rarely applied to LED thermal design but only applied to electronic devices whose outer surface heat flux density does not exceed $10 \mathrm{~W} / \mathrm{cm}^{2}$ due to its complex system, heavy noise, low reliability, expensive maintenance costs and extra power dissipation.

Pool boiling, also known as large container boiling, which is one of liquid boiling methods in industry, is to immerse the heating surface into liquid, and the liquid heats on the wall surface. Pool boiling is greatly valuable in the field of industrial application. In the past few decades, the scholars in different countries have done a lot of research by conducting many boiling heat transfer experiments and put forward various heat transfer mechanism models, but due to the limitations of experiments and assumptions, these models have their own defects [7].

Water phase change cooling, which is based on physical phase change, can only provide $67 \mathrm{~kJ} / \mathrm{mol}$ of heat transfer capacity; the most commonly used gravity heat pipe can only provide $<30 \mathrm{~W} / \mathrm{cm}^{2}$ of heat transfer capacity.

In some extreme physical situations, such as super high temperature reaction kettle, super high temperature gas turbine engine, super mach number propeller, high power laser pump and so on, physical heat sink cooling methods [8] based on physical phase change of working medium are limited by the latent heat of vaporization ability and unable to meet the requirement of heat removal due to high heat flux density. Therefore, since the 1960s, the United States has into practice the study of chemical heat sink application as cooling method [9]. Many studies focused on how to make the chemical endothermic reaction applied to heatdissipation problem [10-12].

The reaction of ethanol dehydration to ethylene is an endothermic reaction with good thermal effect. In the preparation of ethylene, by using the adiabatic tube reactor, heat could be removed from the reactor with the endothermic reaction, while coke formation is avoided.

Through endothermic reaction, heat could be removed from a low temperature heat source, and through exothermic reaction, heat could be upgraded to heat sink at a high temperature. Karaca et al. [13] studied the chemical heat pump system made up with methanol-formaldehydehydrogen, ethanol-acetaldehyde-hydrogen, propanol-acetone-hydrogen, butanol-butyraldehyde-hydrogen and so on at a low temperature. In the system, dehydrogenation is endothermic while hydrogenation is exothermic. On the basis of economic analysis, a large amount of waste heat is greatly needed to achieve the thermal efficiency.

A new chemical approach, which use $\mathrm{C}-\mathrm{CO}_{2}$ endothermic reaction applied to instantaneous heat removal in a high heat flux density situation, is put forward in this paper, which is rarely reported currently at home and abroad. Li et al. [14] explored the effect of $\mathrm{CO}_{2}$ on hard coke degradation reaction, but they did not study the method of application of $\mathrm{C}-\mathrm{CO}_{2}$ endothermic reaction to instantaneous heat removal. In this paper, both theoretical calculation and numerical simulation methods are applied to the study of $\mathrm{C}-\mathrm{CO}_{2}$ endothermic chemical reaction. Both of the results showed that $\mathrm{C}-\mathrm{CO}_{2}$ endothermic chemical reaction could help realize instantaneous heat removal under high heat flux.

\section{Theoretical calculation}

The aim of this study is going to verify that instantaneous heat removal can be realized by $\mathrm{C}-\mathrm{CO}_{2}$ endothermic reaction. In the study, activated carbon particles and carbon dioxide gas are used as reactants. As the reaction proceeds, high energy renewable material-CO would be produced. $\mathrm{CO}$ could be used as supplementary medium of power 
source, providing energy through combustion exothermic reaction, the product- $\mathrm{CO}_{2}$ could be used again as a reactant of endothermic reaction. Thus a material cycle will form through above process. Because of the strong heat absorption capacity and the special relationship between reactants and products, this thinking will help realize instantaneous heat removal and material cycle.

Wood activated carbon is to be used in a subsequent experiment, whose specific surface area is more than $1,000 \mathrm{~m}^{2} / \mathrm{g}$. Thus $\mathrm{CO}_{2}$ can diffuse to internal of activated carbon particle fully for its porosity, which means activated carbon particles will react with $\mathrm{CO}_{2}$ directly. Therefore, the influence of diffusion on reaction rate can be neglected.

$\mathrm{C}-\mathrm{CO}_{2}$ reaction can be simplified as follows [15]:

$\mathrm{CO}_{2}+\mathrm{C} \stackrel{\kappa_{1}}{\longrightarrow} \mathrm{CO}+[\mathrm{CO}]$

$[\mathrm{CO}] \stackrel{\kappa_{2}}{\longrightarrow} \mathrm{CO}$

According to solid surface reaction model, two rate equations can be concluded:

$\frac{d P_{C O}}{d t}=\kappa_{1} P_{C O_{2}}(1-\theta)+\kappa_{2} \theta$

$\frac{d \theta}{d t}=\frac{1}{\beta}\left[\kappa_{1} P_{C O_{2}}(1-\theta)-\kappa_{2} \theta\right]$

In the equations, $P_{\mathrm{CO}}$ and $\mathrm{P}_{\mathrm{CO}_{2}}$ refers to partial pressures of $\mathrm{CO}$ and $\mathrm{CO}_{2}, \kappa_{1}$ and $\kappa_{2}$ are the reaction rate constant of reaction (A) and (B), $\theta$ is to refer to surface fraction occupied by surface oxide [CO]. $\beta$ means absorption capacity.

At a high temperature, $\mathrm{C}-\mathrm{CO}_{2}$ reaction can be considered as first-order reaction, and starting from $800{ }^{\circ} \mathrm{C}$, order of reaction will be close to 1 . The higher the temperature is, the closer to the first-order reaction. A conclusion can be drawn from the above two equations. The higher the temperature is, the $\theta$ is closer to 0 . Then reaction rate is: $\frac{d P_{C O}}{d t}=\kappa_{2} P_{C_{2}}$, which means reaction rate is proportional to $P_{\mathrm{CO}_{2}}(n=1)$; the lower the temperature is, the $\theta$ is closer to 1 . Then the reaction rate is: $\frac{d P_{c O}}{d t}=\kappa_{2} \theta$, which means the reaction rate is independent of $P_{\mathrm{CO}_{2}}(n=0)$.

$\mathrm{C}-\mathrm{CO}_{2}$ reaction is a strong endothermic one, its temperature produces a slight effect on activation energy. Table 1 shows the pre-exponential factor $A$ and reaction activation energy $E a$ [16].

According to Arrhenius formula and chemical reaction rate equation,

Table 1 Reaction parameters

\begin{tabular}{lll}
\hline Reaction & $\begin{array}{l}\text { Pre-exponential factor } \\
A / 1 / \mathrm{s}\end{array}$ & $\begin{array}{l}\text { Activation energy } E a l \\
(\mathrm{~kJ} / \mathrm{mol})\end{array}$ \\
\hline $\mathrm{CO}_{2}+\mathrm{C}=2 \mathrm{CO}$ & $5.55 \times 10^{3}$ & 306.1 \\
\hline
\end{tabular}

$\kappa=A e^{-\frac{E a}{R T}}$

$\gamma=\kappa \cdot c$

Time for removing $500 \mathrm{~W} / \mathrm{cm}^{2}$ heat at different temperatures can be calculated. In the reaction, $\mathrm{CO}_{2}$ concentration is used.

Table 2 and Fig. 1 show time for removing $500 \mathrm{~W} / \mathrm{cm}^{2}$ heat at different temperatures with $\mathrm{C}-\mathrm{CO}_{2}$ endothermic reaction.

From Table 2, it can be seen that, the higher a reaction temperature is, the less time it needs. Time for completing the endothermic reaction can reach the level of one percent second at a certain temperature (about 1,573 K).

To illustrate the strong endothermic capacity of $\mathrm{C}-\mathrm{CO}_{2}$ reaction, a comparison can be made between heat removal capacity of $\mathrm{C}-\mathrm{CO}_{2}$ endothermic reaction and water phase change under the same conditions (the same temperature, pressure and molar amount).

Air density is $1.225 \mathrm{~kg} / \mathrm{m}^{3}$, heat capacity is $1.006 \mathrm{~kJ} /$ $\mathrm{kg}{ }^{\circ} \mathrm{C}$ under constant pressure (from FLUENT database). Length of cylindrical reactor used in numerical simulation is $2 \mathrm{~m}$, and its bottom radius is $0.225 \mathrm{~m}$. If hot air is cooled from 1,500 to $300 \mathrm{~K}$, heat to be removed is $470.35 \mathrm{~kJ}$. Reaction equations of $\mathrm{C}-\mathrm{CO}_{2}$ reaction and water phase change are as follows:

Table 2 Relationship between reaction temperature and time \begin{tabular}{llllllll}
\hline Temperature/ & 973 & 1,073 & 1,173 & 1,273 & 1,373 & 1,473 & 1,573
\end{tabular}

$\mathrm{K}$

\begin{tabular}{llllllll}
\hline Time/s & 23 & 6.37 & 2 & 0.78 & 0.37 & 0.17 & 0.09
\end{tabular}

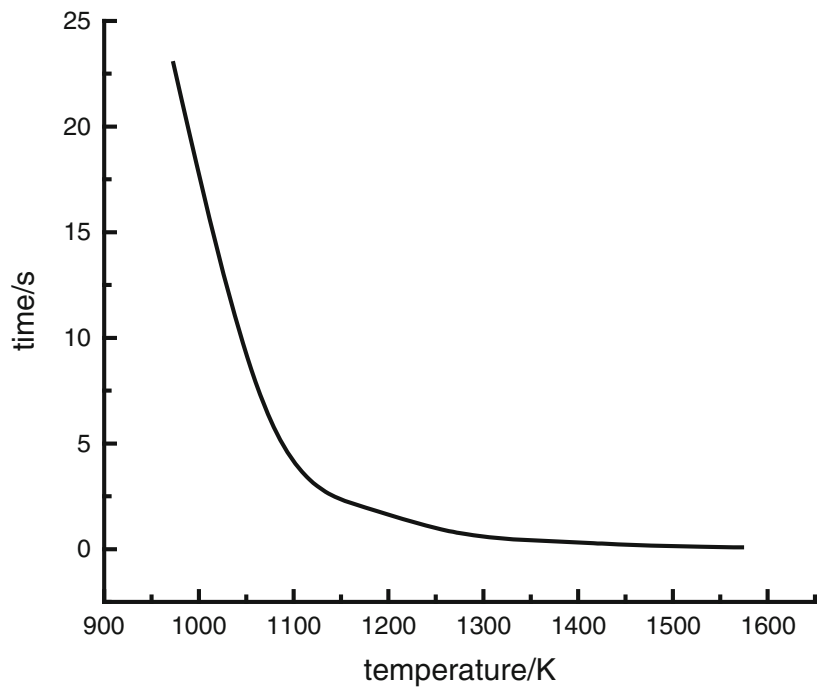

Fig. 1 The graph of time variation with reaction temperatures 
$\mathrm{C}+\mathrm{CO}_{2} \rightarrow 2 \mathrm{CO} ; \quad \mathrm{Q}=172.5 \mathrm{~kJ} / \mathrm{mol}$

$\mathrm{H}_{2} \mathrm{O}(\mathrm{l}) \rightarrow \mathrm{H}_{2} \mathrm{O}(\mathrm{g}) ; \quad \mathrm{Q}=67 \mathrm{~kJ} / \mathrm{mol}$

If $\mathrm{Q}$ is of positive value, the reaction is endothermic. To remove $470.35 \mathrm{~kJ}$ heat, $\mathrm{CO}_{2}$ needed is $2.73 \mathrm{~mol}$, while only $182.7 \mathrm{~kJ}$ heat can be removed through water phase change if the amount of molar water is the same, which means the temperature of hot air can only be cooled to $1,034 \mathrm{~K}$. In comparison of heat absorption capacity of $\mathrm{C}-\mathrm{CO}_{2}$ reaction with water phase change, the former is much stronger than the latter. At the same time, the calorific value of $\mathrm{CO}$, the product, is about $6 \%$ higher, which is equivalent to transfer energy from one medium to the other. $\mathrm{CO}$ can be used as a high calorific value fuel for cycle, which provides necessary conditions for energy cycle.

\section{Numerical simulation}

\subsection{Governing equations}

An axial-symmetrical two-dimensional ( $\mathrm{x}-\mathrm{y})$ computational domain is profiled as shown in Fig. 2, which includes two flow streams. The model has the following dimensions: Diameter of inlet- 1 and inlet-2 are 0.01 and $0.45 \mathrm{~m}$, respectively; thickness of the sheet is $0.005 \mathrm{~m}$.

The following governing equations, Eqs. (5)-(10), for mass, momentum, energy and species transfer are universally applicable to the entire computational domain. However, zero velocities need to be assigned to the solid area in the numerical treatment.

Mass equation [17]:

$\frac{\partial \rho}{\partial t}+\operatorname{div}(\rho U)=0$

Momentum equation [18]:

$\frac{\partial(\rho u)}{\partial x}+\frac{1}{y} \frac{\partial(y \rho v)}{\partial y}=0$

$$
\begin{aligned}
\frac{\partial(\rho u u)}{\partial x}+\frac{1}{y} \frac{\partial(y \rho u v)}{\partial y}= & -\frac{\partial P}{\partial x}+\frac{\partial}{\partial x}\left(\mu \frac{\partial v}{\partial x}\right)+\frac{1}{y} \frac{\partial}{\partial y}\left(y \mu \frac{\partial u}{\partial y}\right) \\
& +\frac{\partial}{\partial x}\left(\mu \frac{\partial u}{\partial x}\right)+\frac{1}{y} \frac{\partial}{\partial y}\left(y \mu \frac{\partial v}{\partial x}\right) \\
\frac{\partial(\rho u v)}{\partial x}+\frac{1}{y} \frac{\partial(y \rho v v)}{\partial y}= & -\frac{\partial P}{\partial y}+\frac{\partial}{\partial x}\left(\mu \frac{\partial v}{\partial x}\right)+\frac{1}{y} \frac{\partial}{\partial y}\left(y \mu \frac{\partial u}{\partial y}\right) \\
& +\frac{\partial}{\partial x}\left(\mu \frac{\partial u}{\partial y}\right)+\frac{1}{y} \frac{\partial}{\partial y}\left(y \mu \frac{\partial v}{\partial y}\right)-\frac{2 \mu v}{y^{2}}
\end{aligned}
$$

Energy equation [18]:

$$
\begin{aligned}
\frac{\partial\left(\rho C_{p} u T\right)}{\partial x}+\frac{1}{y} \frac{\partial\left(y p C_{p} v T\right)}{\partial y}= & \frac{\partial}{\partial x}\left(\lambda \frac{\partial T}{\partial x}\right)+\frac{1}{y} \frac{\partial}{\partial y}\left(y \lambda \frac{\partial T}{\partial y}\right) \\
& +q
\end{aligned}
$$

Species transport equation [18]:

$$
\begin{aligned}
\frac{\partial\left(\rho u Y_{I}\right)}{\partial x}+\frac{1}{y} \frac{\partial\left(y \rho v Y_{I}\right)}{\partial y}= & \frac{\partial}{\partial x}\left(\rho D_{I, m} \frac{\partial Y_{I}}{\partial x}\right) \\
& +\frac{1}{y} \frac{\partial}{\partial y}\left(y \rho D_{I, m} \frac{\partial Y_{I}}{\partial y}\right)+S_{m}
\end{aligned}
$$

The energy equation applied to the solid components of the computational domain reduces to a heat conduction equation since zero velocity is assigned there. Heat generation rates are introduced into the source terms of Eq. (9).

\subsection{Boundary conditions}

Simulations are performed using the FLUENT [19] code. The boundary conditions for the momentum, heat and mass conservation equations are as follows. On the surface of wall, uniform heat flux is utilized to indicate heat source. The model is set to "symmetric" to account for the symmetrical computational domain. The wall is specified as "heat flux" with $500 \mathrm{~W} / \mathrm{cm}^{2}$. Interior side of the wall is set as "coupled wall". The velocity of inlet- 1 and inlet- 2 is set to 2 and $10 \mathrm{~m} / \mathrm{s}$,
Fig. 2 The symmetrical geometric plane structure of the reactor

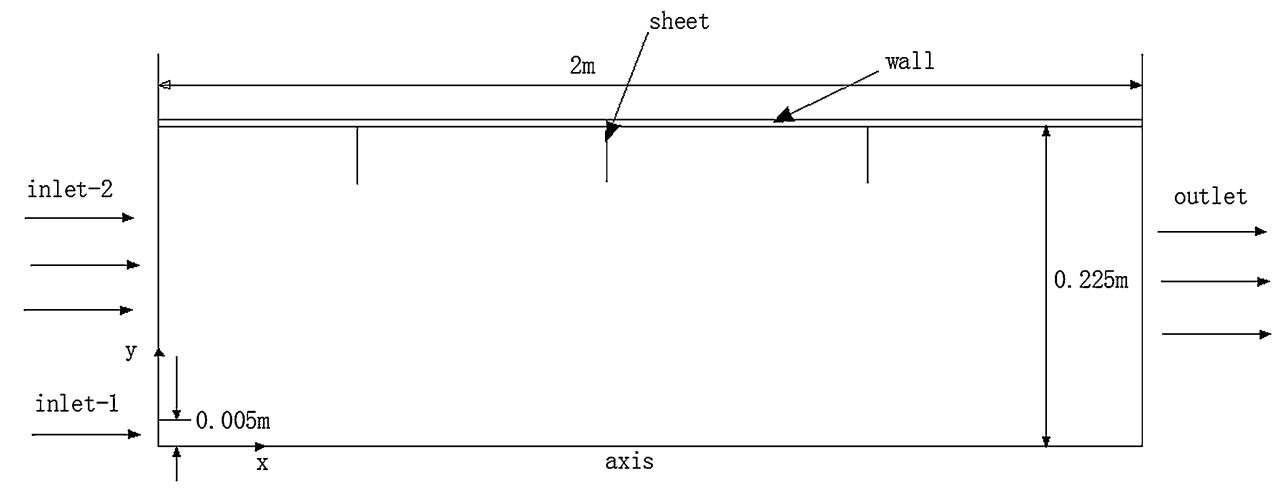


respectively. A "pressure outlet" boundary condition is set at the outlet. The reactants adopted are activated carbon particles and mixture of $\mathrm{CO}_{2}-\mathrm{H}_{2} \mathrm{O}(\mathrm{g})$, respectively. In the mixture, water vapor is used as inert gas, and mass fraction of carbon dioxide is 0.4 . Activated carbon particles enter the reactor from inlet-1, while the mixture enters from inlet- 2 . Three thin pieces of sheets are set for the reactants to mix each other better and at the same time, the model is divided into four parts. And then pressure-based solver is selected. Through calculation, flows of activated carbon particles and $\mathrm{CO}_{2}$ gas belong to turbulent flow. Therefore, k-epsilon ( 2 eqn) standard turbulent model is adopted and then standard wall surface function is used to simulate gas flow. All the above governing equations are discretised by using the finite volume approach and the SIMPLE algorithm is adopted to treat the coupling of the velocity and pressure fields. The first order upwind scheme is selected for the solution to the momentum and energy equations.

\subsection{Results and discussion}

\subsubsection{Heat and mass transfer under high heat flux}

The minimum temperature is $973.9 \mathrm{~K}$ when $\mathrm{C}-\mathrm{CO}_{2}$ reaction begins [14]. Temperature in the reactor is about $1,500 \mathrm{~K}$ before reaction begins, which means the reaction can occur. Figure $3 \mathrm{a}$ refers to the temperature field after $\mathrm{C}-\mathrm{CO}_{2}$ endothermic reaction is completed and Fig. $3 \mathrm{~b}$ is an average temperature distribution graph corresponding to
Fig. 3a. Figure 4a is concerned with $\mathrm{CO}$ mass fraction field and Fig. $4 \mathrm{~b}$ is relevant to $\mathrm{CO}$ average mass fraction distribution graph corresponding to Fig. 4a. These figures and graphs are extracted under these conditions: velocity of $\mathrm{CO}_{2}$ is $10 \mathrm{~m} / \mathrm{s}$, velocity of activated carbon particles is $2 \mathrm{~m} / \mathrm{s}$, and operating pressure is $101.325 \mathrm{kPa}$.

It can be seen from Fig. 3a, b that temperature in the reactor drops obviously after $\mathrm{C}-\mathrm{CO}_{2}$ endothermic reaction is completed. The maximum temperature is just about $330 \mathrm{~K}$ and average temperature is $222 \mathrm{~K}$. Temperature is much lower, which is below average temperature at the first part of model, and this means that the purpose of lowering temperature has come true.

Heat that has been removed and $\mathrm{CO}_{2}$ that has been used in the process can be calculated. Mixture of $\mathrm{CO}_{2}$ and water vapor is cooled from 1,500 to $222 \mathrm{~K}$, according to the endothermic equation below:

$Q=C_{p} m \Delta T$

$Q$ means endothermic or exothermic. In the case, $C_{p}$ is a equivalent value of $\mathrm{C}_{\mathrm{pCO}_{2}}$ and $\mathrm{C}_{\mathrm{pH}_{2} \mathrm{O}(\mathrm{l})}, 1,427.185 \mathrm{~J} /(\mathrm{kg} \mathrm{K}) ; \mathrm{m}$ refers to the mass of mixture: $0.333 \mathrm{~kg} ; \Delta T$ stands for temperature change: $1,278 \mathrm{~K}$. Therefore, heat that has been removed is $607.37 \mathrm{~kJ}$; molar number of $\mathrm{CO}_{2}$ is 3.52 . If the same amount of water is used to remove the heat by water phase change, only $235.9 \mathrm{~kJ}$ heat will be removed, which means mixture of $\mathrm{CO}_{2}$ and water vapor will drop to $1,003.6 \mathrm{~K}$.

It can be found from Figs. 3 and 4 that temperature field and $\mathrm{CO}$ mass fraction field have the same distribution trend:
Fig. 3 a Contours of temperature and $\mathbf{b}$ the distribution graph of average temperature with position on the symmetry plane of the reactor
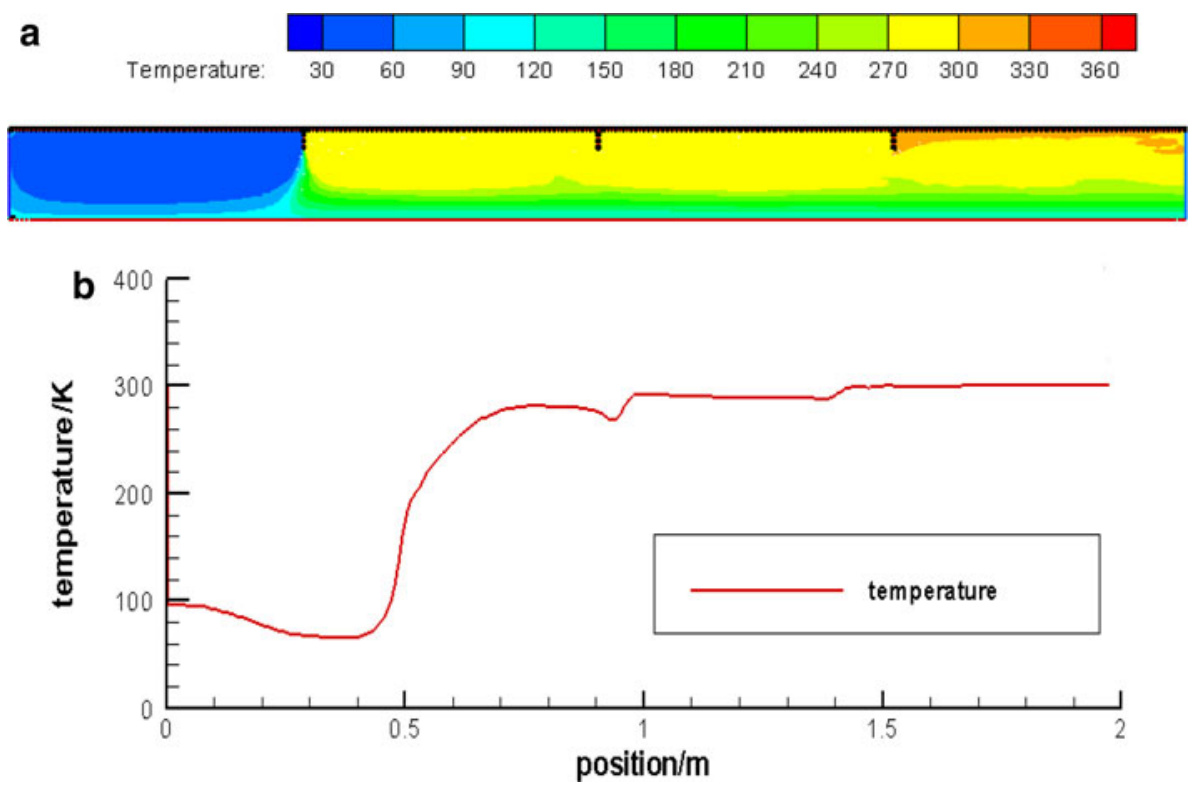
Fig. 4 a Contours of CO mass fraction and $\mathbf{b}$ the distribution graph of average CO mass fraction with position on the symmetry plane of the reactor
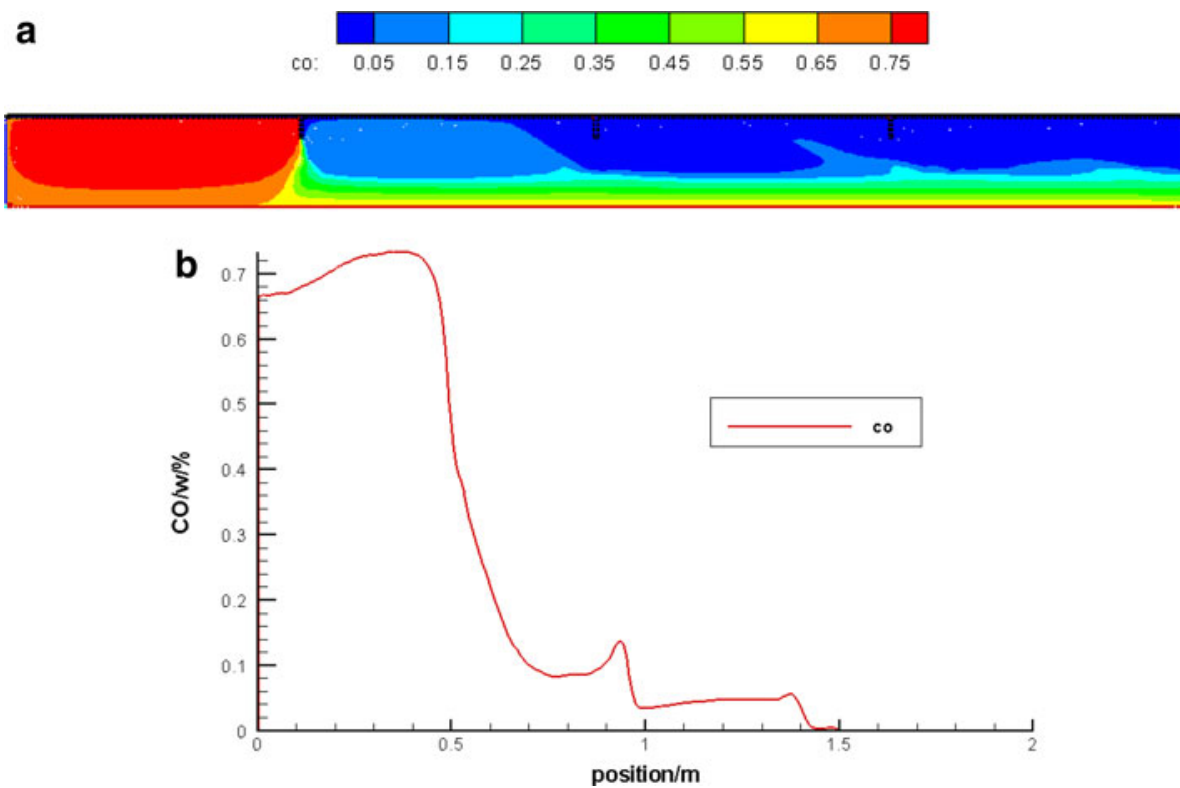

In the first part of model, CO mass fraction is highest and temperature is lowest; in other parts, $\mathrm{CO}$ mass fraction is lower and temperature is higher than that of the first part. Because the inlets of reactants are in the first part, then the amount of $\mathrm{CO}_{2}$ gas and activated carbon particles is larger, which makes them mix more fully and react more completely. $\mathrm{CO}_{2}$ gas and activated carbon particles need to be filled into the area constantly to keep the reaction moving, so that the amount of $\mathrm{CO}_{2}$ gas and activated carbon particles flowing into the rest parts is less and less. Later, the less the amount is, the more slightly the reaction is, so the temperature does not drop so low as that of the first part does. It can be seen from Fig. 4 that $\mathrm{CO}$ mass fraction is almost 0 in the fourth part of model, corresponding to Fig. 3, there is a small high temperature area in the fourth part. In spite of the fact that except the first part of the model, the reaction proceeds more and more slowly and slightly in other parts. Because of the drastic endothermic reaction in the first part, heat from the rest parts transfers to this part, which results in the drop of temperature in other parts.

\subsubsection{Instantaneous heat removal}

Through the calculation above, $607.37 \mathrm{~kJ}$ heat need to be removed for cooling mixture of $\mathrm{CO}_{2}$ and water vapor from 1,500 to $222 \mathrm{~K}$ by employing $\mathrm{C}-\mathrm{CO}_{2}$ endothermic reaction, $3.52 \mathrm{~mol} \mathrm{CO}_{2}$ will be consumed in the process. The results of numerical simulation show that average mass fraction of $\mathrm{CO}_{2}$ reaches $8.1 \times 10^{-05}$ at the end of reaction. In comparison with the mass fraction 0.4 prior to reaction, it is insignificant. So it can be considered that $\mathrm{CO}_{2}$ reacts completely. From Figs. 3 and 4, it is known that after $\mathrm{CO}_{2}$ gas and activated carbon particles enter the reactor, they react at a high temperature condition. According to the data of numerical simulation, time for removing $500 \mathrm{~W} / \mathrm{cm}^{2}$ heat at temperature of $1,500 \mathrm{~K}$ needs $0.142 \mathrm{~s}$. The result proves that the destination of instantaneous heat removal can be realised if $\mathrm{C}-\mathrm{CO}_{2}$ endothermic reaction is used, because the temperature in the reactor drops to such a low level during such a short time.

\subsubsection{Validation of numerical study}

In the part of theoretical calculation, heat to be removed was calculated when temperature dropped from 1,500 to $300 \mathrm{~K}$ by cooling by means of $\mathrm{C}-\mathrm{CO}_{2}$ endothermic reaction, which was $470.35 \mathrm{~kJ}$. And in the numerical simulation, temperature was cooled from 1,500 to $222 \mathrm{~K}$, heat to be removed was $607.37 \mathrm{~kJ}$. Compared with the theoretical results, it is reasonable and receivable. Table 2 shows time for removing $500 \mathrm{~W} / \mathrm{cm}^{2}$ heat at different temperatures. When temperatures are 1,473 and $1,573 \mathrm{~K}$, it takes 0.17 and $0.09 \mathrm{~s}$, respectively. In the numerical simulation, the highest temperature in the model was $1,500 \mathrm{~K}$, and the reaction time could be calculated, $0.142 \mathrm{~s}$, which agreed with the law of temperature and time.

\section{Conclusion}

In this study, $\mathrm{C}-\mathrm{CO}_{2}$ chemical endothermic reaction process is studied by means of theoretical calculation and 
numerical simulation. Theoretical calculation results show that $\mathrm{C}-\mathrm{CO}_{2}$ chemical endothermic reaction can possibly remove heat enormously and instantaneously under a high heat flux density situation. The results of numerical simulation agree with theoretical calculation. Both of the results show that $\mathrm{C}-\mathrm{CO}_{2}$ chemical endothermic reaction has stronger heat absorption capacity than water phase change (about 2.57 times) does, which is a typical physical heat sink. It takes less time to remove the same heat. In other words, using a small amount of $\mathrm{C}$ and $\mathrm{CO}_{2}$ can have the effect of instantaneous and large heat removal, instead of using a large amount of water. At the same time, the product $\mathrm{CO}$ can be used as supplementary medium of power source, providing energy by combustion exothermic reaction. $\mathrm{CO}_{2}$, caused by the exothermic reaction, can also be used as a reactant of endothermic reaction. Thus the above process can form a material cycle. In this way, instantaneous heat removal and a material cycle are realized simultaneously.

Open Access This article is distributed under the terms of the Creative Commons Attribution License which permits any use, distribution, and reproduction in any medium, provided the original author(s) and the source are credited.

\section{References}

1. Chen X (1998) Erosion on solid electrode surface caused by high density electric arc effect. High Volt Apparatus 4:48-50

2. Luo M, Jiang J, Chang A, Zhao D (2004) Study on mechanism of electrode erosion of high-power gas spark gap switch. High Power Laser Part Beams 16(6):781-786
3. Han Y, Wang X, Qiu L, Yu M, Zhao S, Jia W, Ai S (2010) Numerical study on heat transfer characteristics of jet array impingement. Turbine Technol 52(3):161-164

4. Tian C, Xu H, Cao H, Si C (2009) Cooling technology for highpower solid-state laser. Chin J Lasers 36(7):1686-1690

5. He F, Mi Z, Sun H (2006) Improvement of heat sink of endothermic hydrocarbon fuels. Prog Chem 18(7/8):1041-1047

6. Huang M (2006) Thermal degine of the forced air-cooling radar cabinet. Electro-Mech Eng 22(2):12-13

7. Xiao B-Q et al (2009) Mathematical analysis of pool boiling heat transfer. Acta Phys Sin 58(4):2523-2527

8. Yang J, Chou LC, Pais MR (1996) Nucleate boiling heat transfer in spray cooling. J Heat Transf 118:668-671

9. Bland RB et al. (1962) US3067594, Cooling with endothermic chemical reactions

10. Wessberg S, Rasmussen JN (2007) (Carlsberg Brewery Co., Ltd.). CN200780024566.6, Chemical cooling

11. Guo H-b (J\&R Fire Fighting Group). (2010) CN201010013619.8, Chemical coolant for hot aerosol catalyst and preparation method

12. Liu J, Shao J, Liu C, Rao H, Li Z, Li X (2010) Pyrolysis mechanism of hydrocarbon fuels and kinetic modeling. Acta Chim Sin 68(3):239-245

13. Karaca $F$ et al (2002) Economic analysis and comparison of chemical heat pump systems. Appl Therm Eng 22:1789-1799

14. Li J, Lu K, Wang J, Wang $\mathrm{P}$ (2008) Influence of $\mathrm{H}_{2} \mathrm{O}-\mathrm{CO}_{2}$ gas mixture on coke degradation. $\mathbf{J}$ Anhui Univ Technol 25(3):233-236

15. Chen $\mathrm{Z}$ (1984) Study on kinetic and mechanism of $\mathrm{CO}_{2}-\mathrm{C}$ reaction. Tech Equip Environ Pollut Control 5(1):45-49

16. Cen K, Yao Q, Cao X et al (1997) Theory and application of combustion, flow, heat transfer, gasification of coal slurry. Zhe Jiang University Press, Hangzhou

17. Patankar SV (1980) Numerical heat transfer and fluid flow. McGraw-Hill, New York

18. Li P-W, Chyu MK (2003) Simulation of the chemical/electrochemical reactions and heat/mass transfer for a tubular SOFC in a stack. J Power Sources 124:487-498

19. FLUENT 6 User's Guide, Fluent Inc, Lebanon, NH, 2000 